\title{
Standard Methods and Reference Materials for Performing a Complete EPMA WDS Instrument Diagnostic
}

\author{
D. C. Meier, E. S. Windsor, J. M. Davis, R. B. Marinenko, J. R. Anderson, F. Meisenkothen, and S. A. \\ Wight
}

Material Measurement Laboratory, National Institute of Standards and Technology, Gaithersburg, MD 20899-8371

An analytical laboratory quality assurance (QA) program should ideally confirm whether or not a given instrument is capable of making the best possible measurement per manufacturer specifications. There are two indispensable components of such a QA program. The first is a designed test analytical procedure for QA that can result in the best possible measurement given ideal conditions. The second is an ensemble of specimens and reference materials upon which the analytical procedure is performed that are suitable for testing the limits of the instrument's capabilities. The first component can be addressed by carefully standardized methods; the second, by carefully designed and analyzed reference materials.

A comprehensive standardized QA method, in the context of electron probe microanalysis (EPMA) wavelength dispersive spectroscopy (WDS), is designed to assure that the function of each independent component of the instrument, including electron emission source, wavelength dispersion elements, photon counters, and spectrometer motion hardware, is tested. Furthermore, the experimental design should test each of these components to the limits of their capability in as efficient a manner possible, so as to maximize the analytical benefit while using minimal laboratory resources. Ongoing development efforts of such a standard, ISO CD 19463, will be discussed in the context of these design limits.

Upon establishing the parameters of a standard QA method, the results of performing this experimental procedure will be demonstrated using NIST mineral glasses K-411 and K-412 from NIST SRM 470. The specific physical properties that make the SRM 470 glasses suitable specimens for QA, such as stability under electron beam and minimal compositional heterogeneity within and between specimens, will be discussed. The results of weekly QA compositional analyses of these glasses using suitable reference materials will be presented using a variety of statistical analysis, such as normality tests, and graphical representations, such as control charts, box plots, and bean plots (Figure 1), in order to demonstrate how actionable information can be extracted from periodic QA measurements.

Finally, the primary limitation of K-411 and K-412 will be identified; specifically, these glasses are not composed of appropriate elements necessary to fully test the functionality of pentaerythritol (PET) or lithium fluoride (LiF) dispersion elements. For this reason, a glass engineered specifically to serve as the test specimen for EPMA WDS QA has been under development. The glass, designated ADM6XXX, is being integrated into a comprehensive QA program that effectively covers the analytical requirements of most of the periodic table, using a single test specimen and reference material specimen ensemble, capable of assessing the functionality of every spectrometer equipped with thallium acid phthalate (TAP), PET, or $\mathrm{LiF}$ dispersion elements on the instrument simultaneously. Under electron beam excitation, this engineered glass emits characteristic X-rays that include first order and second order diffraction lines for two characteristic $K$ lines on each of these three crystals ( $\mathrm{Zn}$ and $\mathrm{Ge}$ on $\mathrm{LiF}, \mathrm{Ca}$ and $\mathrm{Ti}$ on PET, and $\mathrm{Si}$ and $\mathrm{Al}$ on TAP). The standardized QA procedure will be demonstrated analyzing the new materials in order to illustrate the probative value of a more comprehensive QA procedure for EPMA WDS. 


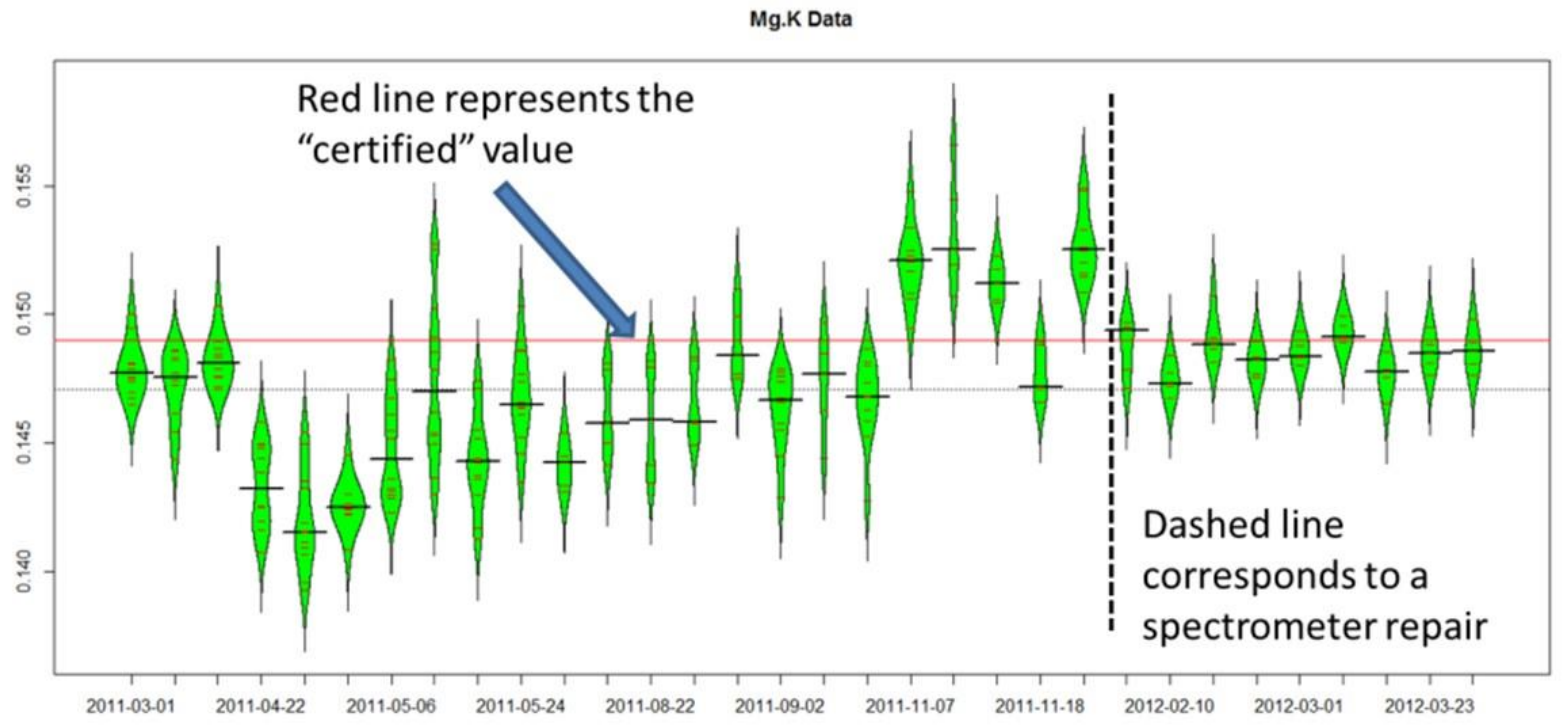

Figure 1. A control chart of bean plots of the Mg composition measurement over a period of months shows performance anomalies that were determined to stem from a fractured TAP crystal. Replacing the crystal (dashed vertical line) reduces the spread and improves the normality of the data.

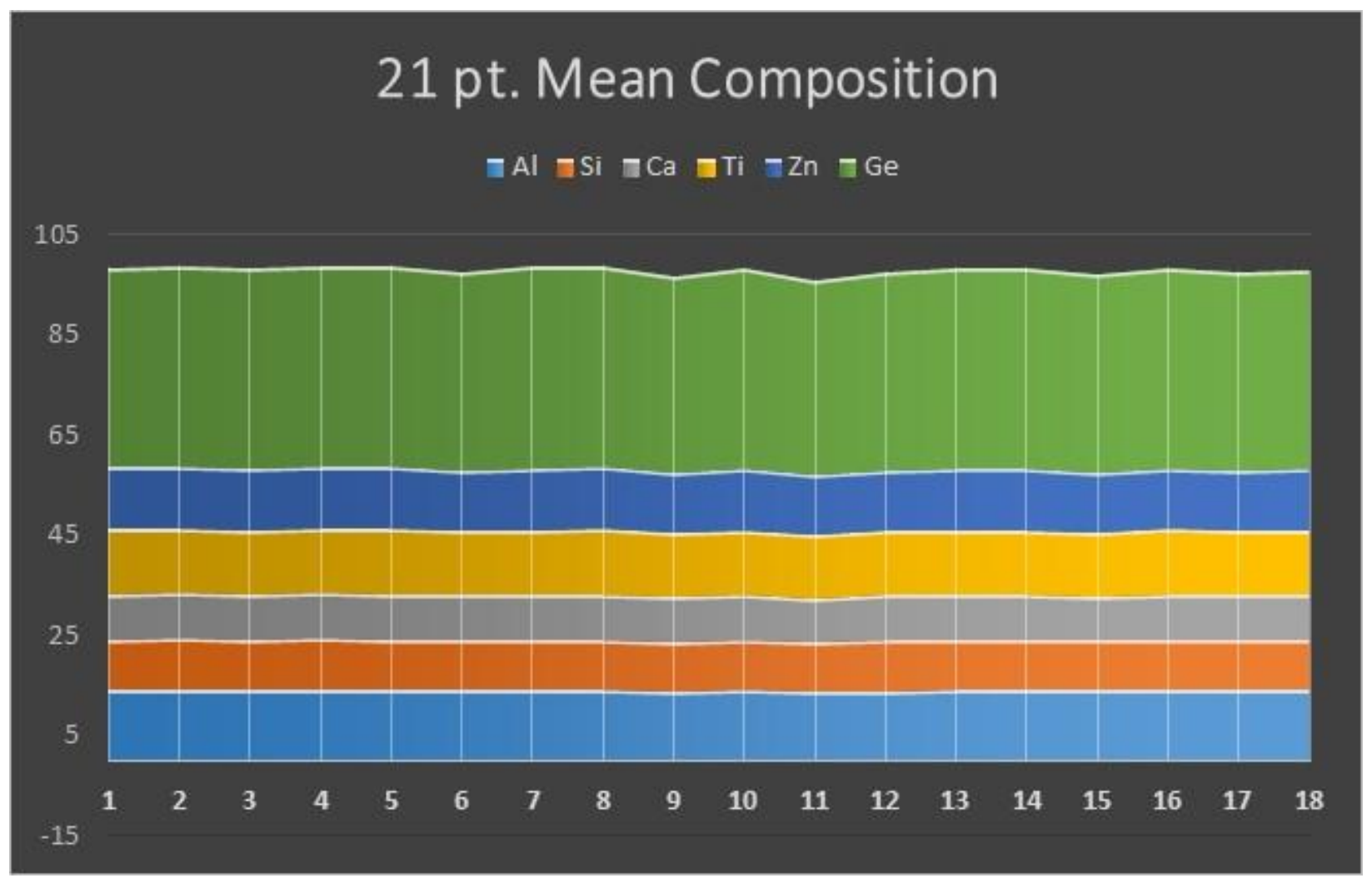

Figure 2. Mean composition of 18 specimens of ADM6XXX glass, confirming heterogeneity is within the compositional resolution of the instrument. 